\title{
43
}

\section{Low temperature opacities}

\author{
C. NEUFORGE
}

Institut d'Astrophysique de l'L'niversité de Liège,5, avenue de Cointe, B-4000 Liège-Belgiu m

\begin{abstract}
The importance of low temperature opacities in stellar calibrations led us to compute new sets of Rosseland mean opacities for different Z-values. For the solar metallicity, these tables have been compared to those of Alexander (1975), Cox (1983), Sharp (1991) and Kurucz (1992).
\end{abstract}

\subsection{Introduction}

Opacities in the atmospheric layers are generally not considered of great importance in the calculation of theoretical evolutionary tracks since the atmosphere of a star only comprises a tiny part of its mass (see however, section 1.2).

Until recently, the most commonly used "atmospheric" or "low-T" opacity tables were those of Cox \&. Stewart (1970), Alexander (1975) and Cox (1983) but there are rather large discrepancies between these different tables for typical $T$ and $\rho$ ranges encountered in stellar atmospheres of solar type stars.

Furthermore, for pop I stars, low-T opacities are calculated for very few values of the metallicity, $Z$, and the solar chemical composition is generally used in the calculation of tracks, whatever the actual value of $\mathrm{Z}$. 


\subsection{Low-T opacities and stellar calibrations}

Theoretical evolutionary tracks depend on mass, age, chemical composition on the zero age main sequence and convection parameter, $\alpha$ (ratio of mixing length to pressure scale height in the convective layers). Calibrating a star consists in computing evolutionary models that reproduce, at given age, chemical composition on ZAMS and convection parameter, the observed values of the luminosity and effective temperature of the star.

It has been shown that uncertainties that may affect low- $T$ opacities (due to missing or wrongly estimated contributors) lead to uncertainties on the convection parameter. Consequently, the calibrated value of $\alpha$ in the Sun actually contains an "opacity component". Unfortunately, problems may be encountered in binary systems, whose components have different effective temperatures. This is the case of the Alpha Centaury system, in which the masses of the two components (alpha Cen $\mathrm{A}$ and $\mathrm{B}$ ) can be determined accurately. Their age $t$ and chemical composition on the ZAMS are assumed to be the same, since both stars have very probably the same origin. Alpha Cen $A$ and $B$ being solar type stars, it seems reasonable to assume that their convection parameter is identical. Then, $\mathrm{Y}, \mathrm{Z}, \mathrm{t}$ and $\alpha$ can, in principle, be adjusted to the two observed luminosities and effective temperatures.

But if the low-T opacities are not known with accuracy, the "opacity component" in $\alpha$ may be different for the two stars (of different Teff) making a calibration in the frame of a unique convection parameter probably inconsistent!

\subsection{New low-T opacity tables}

Problems encountered in stellar calibrations led us to compute new opacity tables for different $Z$-values around the solar metallicity: $Z=0.04,0.03,0.02$ and $X=0.6$ and 0.7 . The tables were computed for $T$ values ranging from 3000 to $15000 \mathrm{~K}$ and for $Q$ values, where $Q=\ln \frac{e^{1 / 3}}{T_{7}}$ (where $T_{7}=\frac{T}{10^{7}}$ ), ranging from -3.838 to 4.605 .

We included the following contributors: HI bf and ff (Karzas and Latter, 1961), $\mathrm{H}^{-}$bf and ff (John, 1988), $\mathrm{H}+\mathrm{H}$ bf (Doyle, 1968), $\mathrm{H}_{2}^{-} \mathrm{ff}$ (Somerville, 1964), $\mathrm{H}_{2}^{+}$bf (Mihalas, 1965), CI, MgI, AlI, SiI, HeI bf and ff (Peach, 1970), $\mathrm{e}^{-}$scattering, $\mathrm{HI}$ and $\mathrm{Hel}$ rayleigh scattering (Dalgarno, 1962), $\mathrm{He}^{-}$ ff (Carbon et al., 1969). Line absorption is included under the form of opacity distribution functions (Gustafsson et al., 1975). Missing UV opacity is also included, according to the relation given by Magain (1983). 

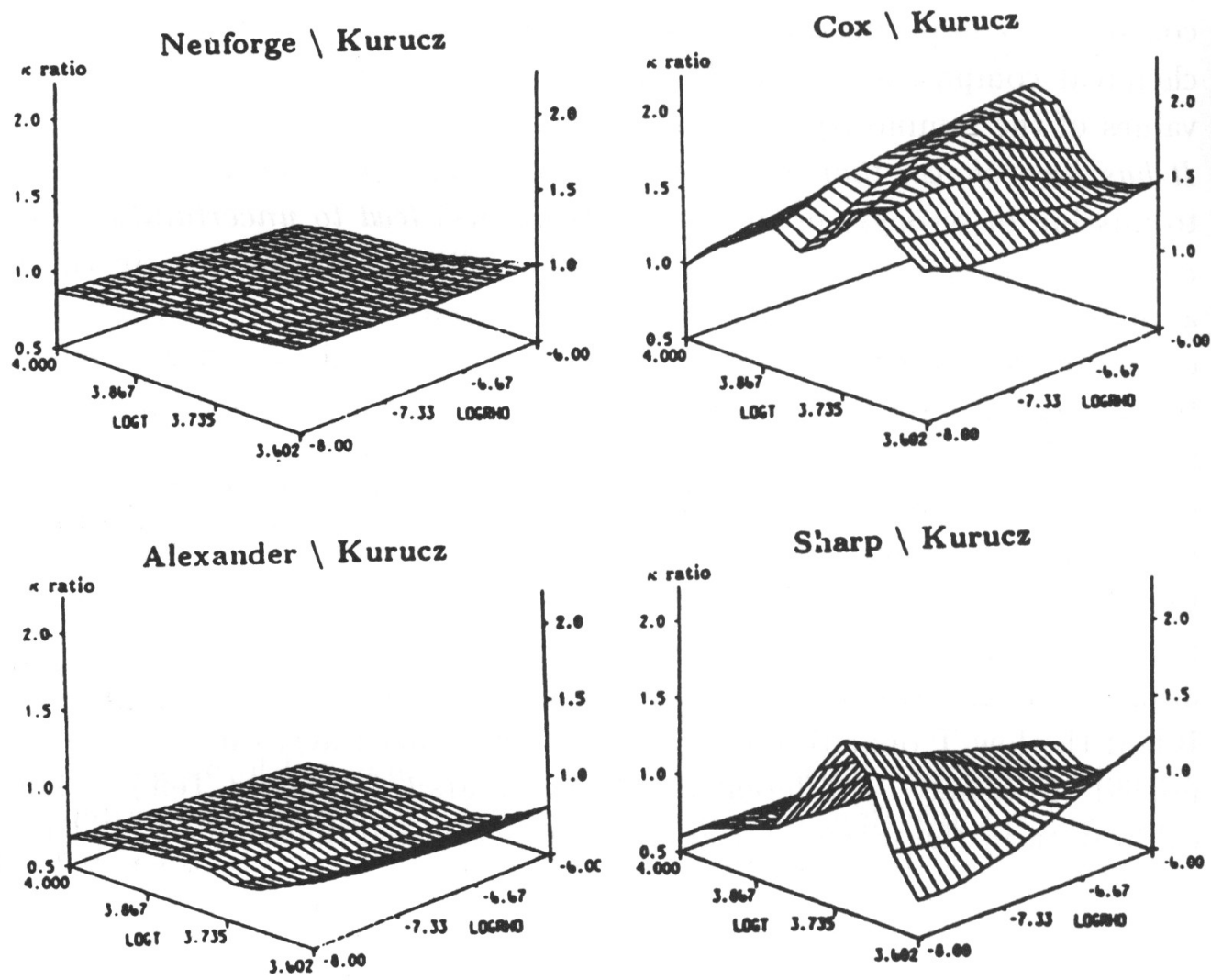

Fig. 43.1 Comparison of our new opacities and the opacities of Alexander (1975), Cox (1983) and Sharp (1991) with those of Kurucz (1992). The ratio of the different opacities are given as a function of $\log T$ and $\log (\rho)$.

\subsection{Comparison with other tables}

For the solar inixture, we compared our results, those of Alexander (1975), Cox (1983) and Sharp (1991) with the results of Kurucz (1992). It is encouraging for us to see that our opacities give the best agreement with those of Kurucz calculations, although we used far less lines than he did. Our re- 
sults remain within less than $13 \%$ of Kurucz's while the other computations show far larger discrepancies.

\subsection{Conclusion}

We have extended our calculations for $\mathrm{X}=0.0,0.2,0.5,0.7$ and 0.9 and $\mathrm{Z}=0.0001,0.001,0.005,0.02$ and 0.04 . For $X<0.5$, UV line absorption is not included, while for $\mathrm{X}=\mathbf{0 . 0}$, only continuous absorption is calculated. These new low temperature opacity tables are available on request (e-mail : U2126CN @ BLIULG11).

\section{References}

Anders, E., Grevesse, N., Gicochim. E' Cosmochim. Acta, 53, 197, (1989).

Alexander, D.R., ApJS, 29, 363, (1975).

Carbon, D., Gingerich, O., Latham, D., "Model Atmosphere for C'ool Dwarf Stars", in "Low Luminosity Stars", Ed S.S. Kumar, Gordon and Breach, New York, (1969).

Cox, A.N., private communication, (1983).

Cox, A.N., Stewart, J.N., Ap.JS., 19, 243, (1970).

Dalgarno, "Spectral Reflectivity of the Earth Atmosphere ", III: "The Scattering of light by Atomic Systems", , GCA Tech. Rep, Ed. Geoph. Corp. of America, (1962).

Doyle, R.O., ApJ, 153, 987, (1968).

Gustafsson, B., Bell, R.A., Erikson, K., Nordlund, $\AA$, ApJ, 42, 407, (1975).

Huebner, W.F., Mertz, A.L., Magee, Jr. N.H., Argo, M.F., Astrophysical Opacity Library, Los Alamos Scientific Lab., Report LA-6760-M, (1977).

John, T.L., $A \& \mathscr{B} A, 193,89$, (1988).

Karzas, W.J., Latter, R. $A p . J S$, 55, VI, 167, (1961).

Kurucz, R.L.,Rev. Mexicana Astron. Astrof., 23, 181, (1992).

Magain, P., $A \& A, 122,225,(1983)$.

Neuforge, C., $A \& A, 268650,(1993)$.

Mihalas, D., ApJS, 92,IV, 321, (1965).

Noels, A., Grevesse, N., Magain, P., Neuforge, C., Baglin, A., Lebreton, Y. A $\mathbb{G}$ $A$, 247, 91, (1991).

Peach, G.,Mem. R. astr. Soc., 73, 1, (1970).

Sackmann, J., Boothroyd, A., Fowler, W. , ApJ, 360, 727, (1990).

Sharp, C., 1991,private: communication, (1991).

Somerville, W.B. ,ApJ, 139, 192, (1964). 